\title{
The NASA/IPAC/NExScI Star And Exoplanet Database
}

\author{
G. B. Berriman*, B. Ali ${ }^{\dagger}$, R. Baker**, K. von Braun ${ }^{\ddagger}$, N-M. Chiu ${ }^{\dagger}$, D. R. \\ Ciardi $^{\ddagger}$, J. Good ${ }^{\dagger}$, S. R. Kane ${ }^{\ddagger}$, M. Kong ${ }^{\ddagger}$, A. C. Laity ${ }^{\dagger}$, D. L. McElroy ${ }^{\dagger}$, \\ S. Monkewitz ${ }^{\dagger}$, A. N. Payne ${ }^{\ddagger}$, S. Ramirez ${ }^{\ddagger}$, M. Schmitz ${ }^{\dagger}$, J. S. Stauffer ${ }^{\S}$ \\ and P. L. Wyatt ${ }^{\ddagger}$ \\ ${ }^{*}$ NASA Exoplanet Science Institute and Infrared Processing and Analysis Center, California \\ Institute of Technology \\ ${ }^{\dagger}$ Infrared Processing and Analysis Center, California Institute of Technology \\ ${ }^{*}$ Raytheon Information Solutions, Pasadena \\ ${ }^{\ddagger}$ NASA Exoplanet Science Institute, California Institute of Technology \\ ${ }^{\S}$ Spitzer Science Center, California Institute of Technology
}

\begin{abstract}
The NASA/IPAC/NExScI Star and Exoplanet Database (NStED) is a general purpose stellar archive which supports NASA planet-finding and planet-characterization goals, stellar astrophysics, and the planning of NASA and other space missions. There are two principal components of NStED: a database of 140,000 nearby stars and exoplanet-hosting stars, and an archive dedicated to high precision photometric surveys for transiting exoplanets (NStED-ETSS). We present summaries of these components. The NStED stellar database currently serves published parameters for 140,000 stars. These parameters include coordinates, multiplicity, proper motion, parallax, spectral type, multiband photometry, radial velocity, metallicity, chromospheric and coronal activity index, rotation velocity/period, infrared excess. NStED-ETSS currently serves data from the TrES survey of the Kepler field as well as dedicated photometric surveys of four stellar clusters. NStED-ETSS aims to serve both the surveys and the broader astronomical community by archiving these data and making them available in a homogeneous format.
\end{abstract}

Keywords: astronomical data bases, catalogs, surveys, time; stars: variables, planetary systems, exoplanets

PACS: $95.80 .+\mathrm{p}$, 97.10.-q, 97.82.-j

\section{INTRODUCTION}

The NASA Star and Exoplanet Database (NStED) is dedicated to collecting and serving vital published data involved in the search for and study of extrasolar planets and their host stars.

NStED consists of two sets of services:

- The stellar and exoplanet services provide access to stellar parameters of potential exoplanet bearing stars along with exoplanet parameters, and

- The Exoplanet Transit Survey Service (ETSS) provides an interface dedicated to searches of exoplanet transit surveys.

Currently, all these services are accessible through simple web forms at the project 
web page 1 . The following sections describe the important features of the stellar and ETSS services.

\section{STELLAR SERVICES IN NSTED}

The stellar services provided by NStED include the following:

- Access to data related to relatively bright nearby stars.

- The capability to display and visualize the properties of individual stars.

- The capability to perform complex searches on stellar and planetary parameters.

- Access to published images, spectra, and time series data related to the stars in the database.

Complementary to these services are the exoplanet services, which include the following:

- Access to general data and pubished parameters for the known exoplanets and host stars.

- Access to, and visualization of, photometric and radial velocity data related to the known exoplanets.

\section{STELLAR CONTENT IN NSTED}

NStED's stellar and exoplanet content is composed of published tabular data, derived and calculated quantities, and associated data including images, spectra, and time series. Some of data sets have been generously contributed by data providers, such as echelle spectra from the N2K consortium ([1]).

NStED's core set of stars is derived from the Hipparcos, Gliese-Jahreiss, and Washington Double Star catalogs. The total number of Hipparcos and Gliese-Jahreiss stars within NStED is approximately 140,000. A summary of the stellar parameters and data within NStED is shown in Table 1. NStED currently supports complex multi-faceted queries on approximately 75 astrophysical stellar and exoplanet parameters.

\section{EXOPLANET CONTENT FOR NSTED}

In order to facilitate future exoplanet studies, NStED maintains an up-to-date list of exoplanetary systems and associated stellar data by monitoring daily the literature and making weekly updates to the database. The predicted signatures of exoplanets are also calculated to aid users in selection of stars appropriate for planet searching and characterization. The exoplanet signature predictions include habitable zone sizes, astrometric and radial velocity wobbles, and transit depths. A summary of the exoplanet parameters and data in NStED is shown in Table 2.

\footnotetext{
${ }^{1}$ http://nsted.ipac.caltech.edu
} 
TABLE 1. Summary of stellar content within NStED.

\begin{tabular}{lll}
\hline Published Parameters & Derived Parameters & Associated Data \\
\hline Position, Distances & Temperature & Images \\
Kinematics & Luminosity & Spectra \\
Photometry, Colors & Radius & \\
Spectral Type & Mass & \\
Luminosity Class & LSR Space Motion & \\
Metallicity & & \\
Rotation & & \\
Activity Indicators & & \\
Variability & & \\
Multiplicity & & \\
\hline
\end{tabular}

TABLE 2. Summary of exoplanet content within NStED.

\begin{tabular}{lll}
\hline Published Parameters & Predicted Parameters & Associated Data \\
\hline Number of Planets & Habitable Zone & High Contrast Images \\
Planetary Mass & Astrometric Wobble & Lightcurves \\
Orbital Period & Radial Velocity Wobble & \\
Orbital semi-major axis & Earth V Magnitude & \\
$\begin{array}{l}\text { Orbital Eccentricity } \\
\text { Link to entry in the }\end{array}$ & Earth $10 \mu$ m flux density & \\
Exoplanet Encyclopaedia & & \\
\hline
\end{tabular}

\section{SPECIFIC GOALS OF NSTED-ETSS}

The purpose of NStED-ETSS is to make available to the astronomical community timeseries light-curves of planet transit studies and other variability surveys in a homogeneous format, along with tools for data analysis and manipulation. The principal goals of NStED-ETSS include the following:

- Provide access to ancillary data for ground-based and space-based transit missions.

- Support optimization of algorithms for transit detection or variability classification on existing survey data sets; for instance, to enable the detection of planets previously missed in the original study.

- Extend the time baseline for transit studies by using data sets containing the same stars, leading to increased detection efficiency, results of increased statistical significance, enhanced potential to conduct transit timing studies, etc.

- Enable improved understanding of false positivies encountered in transit surveys.

- Provide access to a wealth of other astrophysical results and ancillary science not pursued in the original survey, such as studies of eclipsing binary and other variable stars or variability phenomena, stellar atmospheres (rotation, flares, spots, etc.), asteroseismology and intrinsic stellar variability, as well as serendipitous discoveries such as photometric behaviors of supernovae progenitors, etc. 


\section{ETSS HOLDINGS AND FUTURE DATA SETS}

Here we summarize the data sets accessible through ETSS. All the data are organized in a common ASCII format for portability: a master file provides the basic properties of the data set and parameters describing the light curves; there is one light curve file for each star in the data set.

TrES-Lyr1, the TrES network planet transit survey of a field in Lyra, described in [2], contains $\sim 26,000$ stars with 15,500 observation epochs over 75 nights in the $R$ and $r$ filters. The data sets on the globular clusters (GCs) M10 and M12 contain 44,000 and 32,000 stars, respectively, with $\sim 50$ observational epochs in both $V$ and $I$ over a 500night timespan ([3] ). The data set on the GC NGC 3201 features $~ 59,000$ stars with 120 epochs in each $V$ and $I$ over the course of 700 nights $([4,5])$. NGC 2301 is an open cluster and its data set contains 150 epochs in $R$ on 4,000 stars over 14 nights $([6,7])$. The KELT-Praesepe data set [8, 9] contains light curves of 66,637 stars at $R_{K}$ with 3,00 epochs over 73 nights

Data sets will be ingested in 2008 and 2009 include WASP0 (PI: S. R. Kane), VULCAN (PI: N. Batalha), BOKS (PIs: S. Howell \& J. J. Feldmeier), EXPLORE/OC (PIs: $\mathrm{K}$. von Braun \& B. L. Lee), as well as future CoRoT fields, as NStED is collaborating with the CoRoT team to provide a NASA portal to the public CoRoT data [10].

Each featured data set has been graciously donated by the respective survey team. Astronomers wishing to donate data sets are invited to contact the NStED Help Desk 2 .

\section{ACKNOWLEDGMENTS}

The NASA/IPAC/NExScI Star and Exoplanet Database is operated by the Jet Propulsion Laboratory, California Institute of Technology, under contract with the National Aeronautics and Space Administration. NStED wishes to thank the astronomers and research teams who have generously donated their data sets.

\section{REFERENCES}

1. Fischer, D.A., et al. 2005, $A p J, 620,481$

2. O’Donovan, F. T., et al. 2006, ApJ, 651, L61

3. von Braun, K., Mateo, M., Chiboucas, K., Athey, A., \& Hurley-Keller, D. 2002, AJ, 124, 2067

4. von Braun, K., \& Mateo, M. 2001, AJ, 121, 1522

5. von Braun, K., \& Mateo, M. 2002, AJ, 123, 279

6. Howell, S. B., VanOutryve, C., Tonry, J. L., Everett, M. E., \& Schneider, R. 2005, PASP, 117, 1187

7. Tonry, J. L., Howell, S. B., Everett, M. E., Rodney, S. A., Willman, M., \& VanOutryve, C. 2005, PASP, 117,281

8. Pepper, J., et al. 2007, PASP, 119, 923

9. Pepper, J., Stanek, K. Z., Pogge, R. W., Latham, D. W., DePoy, D. L., Siverd, R., Poindexter, S., \& Sivakoff, G. R. 2008, AJ, 135, 907

10. Baglin, A. 2006, in The CoRoT Mission, ESA-SP-1306, 33

${ }^{2}$ http://nsted.ipac.caltech.edu/cgi-bin/Helpdesk/nph-genTicketForm 